1

2

3

4

5

6

7

8

9

10

11

12

13

14

15

16

17

18

19

20

21

22

23

24

25

26

27

28

29

30

31

32

33

34

35

36

37

38

39

40

41

42

43

44

45

46

47

48

49

50

51

52

53

54

55

56

57

58

59

60

Verbal learning and memory following stroke

\title{
Running head: VERBAL LEARNING AND MEMORY FOLLOWING STROKE
}

KEYWORDS: learning and memory; left hemisphere; working memory; picture naming 


\begin{abstract}
Objective. The research examined whether verbal learning and memory impairment previously observed one year after left hemisphere stroke endures over a longer period and whether stroke sufferers compensate for their impairments using working memory. Methodology. Twenty-one persons with left hemisphere lesions; 20 with right hemisphere lesions only and 41 matched controls completed the Hopkins Verbal Learning Test-Revised (HVLT-R), a working memory test (Letter-Number Sequencing, LNS) and the Boston Naming Test (BNT).

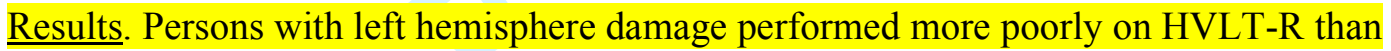
controls. They showed poorer immediate recall, delayed recall, recognition, and learning, but intact retention, suggesting an encoding impairment. BNT and LNS scores predicted recall in this group. HVLT-R performance of persons with right hemisphere lesions only was comparable to controls. BNT (not LNS) predicted recall in these groups.

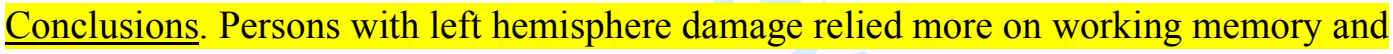
recruited diverse left hemisphere regions to compensate for their impaired encoding. $\underline{\text { Implications. }}$ Tasks requiring verbal encoding and memory are effortful following left hemisphere stroke. This should be recognized and accommodated.
\end{abstract}




\section{Verbal learning and memory following stroke}

The capacity to learn and to remember what we have learnt is fundamental to human functioning. It is often taken for granted until it is lost or impaired following brain damage due to trauma or degeneration. The current research examined verbal learning and memory following stroke. By examining the predictors of verbal learning and memory, we also sought to understand whether people who have suffered a stroke employ the same cognitive processes as unimpaired people (i.e., those associated with regions of the left temporal lobes) or whether they might employ additional processes (i.e., working memory) that recruit more diverse brain regions.

Verbal learning and memory is often assessed using tests such as the Hopkins Verbal Learning Test-Revised (HVLT-R) [1, 2, 3]. In this test, a list of words is read aloud, then the test taker attempts to recall the words in any order. The 12-word list consists of four words from each of three semantic categories (e.g., precious stones, four-legged animals, and dwellings). The same words are presented three times and each presentation is followed by a recall attempt. Learning is indicated by improvement in recall across trials. There is also a delayed free recall test and a recognition test.

Verbal learning and memory in the HVLT-R and similar tests require encoding and consolidation of verbal information for subsequent retrieval. These processes have been shown to depend primarily on regions within the left hemisphere of the brain [4]. The medial temporal lobe (MTL) system has been identified as important for encoding, consolidation and retrieval functions [4]. Frontal regions might also be involved in encoding. A functional imaging study [5] observed predominantly left-lateralised activation in both frontal and MTL regions during encoding of novel verbal stimuli.

The importance of left hemisphere regions in verbal learning and memory is supported by Schouten, Schiemanck, Brand, and Post's [6] research. They found that one 
year after suffering a stroke, patients with left hemisphere lesions $(n=46)$ had impaired immediate and delayed recall on a Dutch version of the Rey Auditory-Verbal Learning Test compared to patients with right hemisphere lesions $(n=40)$. Larger lesions and the presence of subcortical damage were also associated with poorer performance, but there was no significant association with anterior versus posterior location of the lesion. This is consistent with the involvement of diverse regions of the left hemisphere in verbal learning in people who have suffered a stroke.

The current research extends that of Schouten et al. [6] in several ways. First, the current research included unimpaired participants as well as people who had suffered a stroke. Schouten et al.'s research did not include unimpaired participants so it is unclear whether right hemisphere stroke also results in impairment. Second, our research examined whether the impairments evident in Schouten et al.'s patients one year after left hemisphere stroke are also evident after a longer time interval. In order to examine this issue, we examined verbal learning and memory in people who had suffered a stroke in the more distant past. Therefore our patients had had more time to develop strategies to compensate for stroke-related impairments. However, to the extent that recovery is less than complete, impaired verbal learning and memory should be still evident in those who have suffered a left hemisphere stroke.

Third, we also examined two potential predictors of verbal learning and memory: working memory and picture naming, which like the HVLT-R involve encoding and/or retrieval of verbal material. Working memory was assessed using the Letter Number Sequencing (LNS) subtest of the Wechsler Adult Intelligence Scale-third edition (WAIS-III) [7]. In this test, digits and alphabetic letters are presented in a mixed order. Respondents are required to report the digits in ascending order then the letters in alphabetic order. The digits and letters must be encoded and maintained in working memory, while they are sorted and 
then reported in the correct sequence. Thus, encoding, manipulation, and retrieval of verbal material are required. Working memory tasks are thought to rely on networks in the frontal and parietal regions of the brain and there is evidence for left lateralisation of verbal working memory. For a review see [8]. A recent event-related potential study [9] examined the brain regions involved in LNS. In the healthy controls, LNS performance correlated with long interval intra-cortical inhibition over the dorsolateral regions of the prefrontal cortex.

The HVLT-R and LNS both require encoding of verbal material. However, the encoding demands are likely be lower in the HVLT-R because the same conceptually-related words are used on all trials. Repetition of the words and the conceptual relations between them should facilitate encoding. Conceptual relations would also facilitate retrieval [10]. In working memory tasks there is no repetition of stimuli. In the LNS, different letters and numbers are presented on each trial so the stimuli remain novel. This led us to predict that in unimpaired individuals, working memory capacity as assessed in the LNS, would not be a strong predictor of verbal learning and memory as assessed by the HVLT-R.

In the Boston Naming Test (BNT) [11], test takers are required to name common objects that are depicted in line drawings. Among other processes, picture naming requires recognition of the objects and retrieval of lexical-semantic information (object names) from long-term memory. In a voxel-based lesion symptom mapping study of participants who had suffered a single left hemisphere stroke [12], picture naming on the BNT depended on a large network of regions, including portions of the left anterior to posterior middle temporal gyrus and superior temporal gyrus and underlying white matter, as well as inferior parietal cortex. When factors related to speech production and visual perception were controlled, lexicalsemantic retrieval was most dependent on the mid- to posterior portion of the middle temporal gyrus and underlying white matter. 
A process common to verbal learning and memory and to picture naming tasks is retrieval of lexical-semantic information. The HVLT-R requires retrieval of recently encoded, conceptually-related words, whereas the BNT requires retrieval from semantic memory of object names that would have been encoded long ago. To the extent that the retrieval demands are comparable, we might expect picture naming to be a significant predictor of verbal learning and memory in the HVLT-R, at least in individuals with intact left hemispheres.

However the pattern might differ for individuals who have suffered a left hemisphere stroke. Damage to brain regions employed in verbal learning and memory prior to stroke might mean that other less specialised regions are co-opted to help compensate for the damage [13]. In the current study, the time that had passed since stroke occurred was sufficient to allow our participants to develop compensatory strategies. In functional imaging studies of cognitive aging, positive associations are frequently observed between levels of activation in prefrontal cortex and memory performance. Such findings have been interpreted as reflecting compensatory processing whereby older adults rely on strategies mediated by the prefrontal cortex to compensate for declines in processing mediated by more posterior regions [13]. Thus stroke patients with damage to the left hemisphere might employ working memory processes of the prefrontal cortex to encode words in verbal learning tasks such as the HVLT-R. If so, working memory should be a significant predictor of HVLT-R performance. Picture naming should be a significant predictor of HVLT-R performance for the same reasons as in people without left hemisphere damage.

\section{Method}

\section{Participants.}

The sample consisted of 41 people who had suffered a stroke and 41 people who had not. The first language of all participants was English. The unimpaired participants were 
healthy individuals who lived independently in the community. They were recruited through social clubs and sporting groups. The stroke patients were recruited through stroke support groups in south-east Queensland, Australia. Neurologists' reports and MRI/CT scans indicated that 21 patients had left hemisphere damage, 20 had damage to the right hemisphere only. No details related to rehabilitation were collected. However stroke patients in Australia are routinely offered rehabilitation whilst in hospital. The type of rehabilitation (speech therapy, occupational therapy and/or physiotherapy) varies according to the needs of individual patients. The rehabilitation services available following discharge from hospital are more limited. Outpatient clinics of some hospitals provide services, and patients who have health insurance (or the capacity to pay) might choose to access further rehabilitation services privately.

Tables I and II provide further details of the participants. The three groups did not differ significantly in terms of their age, $F(2,79)=0.96, p=.39$, years of education, $F(2$, $79)=0.39, p=.68$, or gender balance, $\chi^{2}(N=82 ; d f=2)=0.07, p=.97$. The two stroke groups did not differ in terms of time since stroke, $F(1,39)=0.07, p=.79$, or the number of patients with damage to anterior regions, $\chi^{2}(N=41 ; d f=1)=1.19, p=.28$, posterior regions $\chi^{2}(N=41 ; d f=1)=0.20, p=.66$, or subcortical regions, $\chi^{2}(N=41 ; d f=1)=3.06, p=.08$.

The National Adult Reading Test [14] was used to assess verbal ability. Participants were required to read a list of 50 words. Each incorrectly pronounced word counted as an error. Error rates were converted to standard scores, which are shown in Table I. ANOVA revealed a significant effect of group, $F(2,76)=8.40, p=.001$, partial $\eta^{2}=.181$. The stroke patients with left hemisphere damage performed significantly more poorly than the unimpaired group $(p=.001)$. The stroke patients with right hemisphere damage only did not differ significantly from the unimpaired group $(p=.121)$ nor the left hemisphere damage $\operatorname{group}(p=.247)$. 


\section{The Picture Completion subtest of the WAIS [7] was included as a marker of} performance ability. Incompletely drawn pictures (e.g., a table with one leg missing) were presented and participants were asked to identify the part that was missing. Participants had 20 seconds to respond to each of the 25 items. The score was the number of correct responses. ANOVA revealed a nonsignificant effect of group, $F(2,79)=2.46, p=.092$, partial $\eta^{2}=.059$. See Table I

Insert Tables I and II here

\section{Measures and Procedures.}

The tasks described below were administered as part of a larger battery, by one of two female research assistants, who had previous experience working with patients with brain injuries. All testing was conducted in the participants' homes. Institutional ethics approval for the research was obtained and all participants provided informed consent.

Hopkins Verbal Learning Test Revised (HVLT-R, Form 1.) [1] is a well-validated measure of verbal learning and memory that has been widely used with clinical samples $[15$, 16]. It consists of a 12-item word list, composed of four words from each of three semantic categories. In Form 1 the categories are precious stones, four-legged mammals, and dwellings. Participants were instructed to listen carefully while the examiner read the words aloud at an approximate rate of one word every 2 seconds. Participants then attempted to recall the words. The same procedure was repeated for two more trials (maximum score $=12$ per trial). The learning index was computed by subtracting the number of words recalled on trial 1 from the highest of trial 2 or trial 3. 
Delayed free recall was assessed after 20 minutes. Participants recalled as many of the 12 words as possible. The retention index was computed by expressing delayed recall as a percentage of trial 3 recall.

The recognition test was presented after the delayed recall test. The examiner read aloud 24 words, twelve of which had been presented previously (targets) and twelve of which had not (distractors). Participants were instructed to say 'yes' after each target word and 'no' after each distractor. The recognition score was the sum of the correct yes and correct no responses (maximum score $=24)$. A discrimination index (maximum score $=12)$ was also computed by subtracting the number of false positives (yes responses to distractors) from the number of true positives (yes responses to targets).

Letter Number Sequencing (LNS) sub-test of the WAIS [7] was used to assess verbal working memory capacity. Mixed lists of letters and numbers (e.g., F - 4 - B - 7) were read aloud by the examiner. List length was two at the outset, and increased by one after every three trials. Respondents were required to first repeat the numbers in ascending order, and then the letters in alphabetical order (e.g., 4-7-B-F). Possible scores ranged from 0 to 21 .

Boston Naming Test (BNT) [11] is a test of confrontation naming. It consists of 60 line-drawings of common objects. Respondents were allowed 20 seconds to name each drawing. Stimulus cues (e.g., the object's function) were offered to correct for misperception errors. When necessary, these were followed by phonemic cues, which provide the first phonemes of the word, facilitating lexical retrieval. The total score on the test was the number of correct responses produced spontaneously and with the aid of stimulus cues (maximum score $=60)$.

\section{Results}


HVLT-R recall scores were subjected to a mixed 3 (Group) $\times 4$ (Trial) ANOVA with trial as the within groups variable with four levels corresponding to trials $1,2,3$ and delayed. Descriptive statistics are shown in Table III. The analysis yielded a significant main effect of group, $F(2,79)=6.17, p=.003$, partial $\eta^{2}=.135$. Planned contrasts showed that mean recall was significantly lower in the stroke group with left hemisphere damage $(6.49 \pm 0.45)$ than in the stroke group with right hemisphere damage only $(7.90 \pm 0.47)(p=.033)$ and also the unimpaired control group $(8.45 \pm 0.33)(p=.001)$. The stroke group with right hemisphere damage only and the unimpaired control groups did not differ significantly $(p=$ $.34)$.

Insert Table III about here

There was a significant main effect of trial, $F(3,237)=88.60, p<.001$ partial $\eta^{2}=$ .529. Repeated measures contrasts showed that recall improved significantly from trial 1 $(5.66 \pm 0.24)$ to trial $2(7.95 \pm 0.27), F(1,79)=132.48, p<.001$, partial $\eta^{2}=.626$, and from trial 2 to trial $3(8.82 \pm 0.26), F(1,79)=34.06, p<.001$, partial $\eta^{2}=.301$, then declined significantly on the delayed recall trial, $(8.02 \pm 0.32), F(1,79)=18.05, p<.001$ partial $\eta^{2}=$ .186.

The main effects were modified by a significant Stroke group $\times$ Trial interaction, $F(6$, $237)=2.48, p=.024$, partial $\eta^{2}=.059$. Stroke group did not interact with the contrast between trials 1 and 2, or with the contrast between trials 3 and delayed, smaller $p=.45$. The interaction of stroke group with the contrast between trials 2 and 3 approached significance, $F(1,79)=2.34, p=.098$, partial $\eta^{2}=.057$. The improvement in recall from trial 2 to trial 3 was significant in the unimpaired control group, $F(1,40)=36.39, p<.001$, partial $\eta^{2}=.476$ and the stroke group with right hemisphere damage only, $F(1,19)=19.48, p<.001$, partial 
$\eta^{2}=.506$, but not in the stroke group with left hemisphere damage, $F(1,20)=2.11, p=.162$, partial $\eta^{2}=.095$. This was the source of the significant Stroke group $\times$ Trial interaction.

The interaction was also partitioned according to trial. The effect of stroke group was not significant on trial $1, F(2,79)=1.91, p=.155$, partial $\eta^{2}=.046$, but it was significant on trial 2, $F(2,79)=3.67, p=.03$, partial $\eta^{2}=.085$, trial $3, F(2,79)=7.85, p=.001$, partial $\eta^{2}$ $=.166$, and the delayed trial, $F(2,79)=6.89, p=.002$, partial $\eta^{2}=.149$. The stroke group with left hemisphere damage recalled significantly fewer words than the unimpaired control group on trial $2(p=.008)$, trial $3(p<.001)$, and the delayed trial $(p<.001)$. The stroke group with right hemisphere damage only did not differ significantly from the unimpaired control group on any trial.

Group differences on additional HVLT-R indices were examined. Descriptive statistics for the learning and retention indices are shown in Table III. For the learning index, ANOVA showed that the overall effect of stroke group approached, but did not reach significance, $F(2,79)=2.51, p=.088$, partial $\eta^{2}=.06$. Planned contrasts showed that there was significantly less learning in the stroke group with left hemisphere damage than in the unimpaired control group, $p=.028$. The unimpaired control group and the stroke group with right hemisphere damage only did not differ significantly $(p=.381)$. For the retention index, ANOVA showed that the overall effect of stroke group did not approach significance, $F(2$, 79) $=0.25, p=.768$, partial $\eta^{2}=.007$

Recognition scores are shown in the rightmost column of Table III. ANOVA revealed that the effect of group approached significance, $F(2,79)=2.63, p=.078$, partial $\eta^{2}$ $=.062$. Planned contrasts showed that recognition accuracy was significantly higher in the control group than in the stroke group with left hemisphere damage $(p=.028)$, but the unimpaired control group and the stroke group with right hemisphere damage only did not differ significantly $(p=.221)$. 
A similar pattern was observed on the discrimination index. ANOVA revealed that the effect of group approached significance, $F(2,79)=2.51, p=.088$, partial $\eta^{2}=.06$. Planned contrasts showed that discrimination was significantly more accurate in the control group than in the stroke group with left hemisphere damage $(p=.035)$, but the control group and the stroke group with right hemisphere damage only did not differ significantly $(p=$ $.189)$.

Predicting HVLT-R performance. Separate regression analyses were conducted for control participants, stroke patients with right hemisphere damage only, and for the stroke patients with left hemisphere damage. In the first set of analyses, the criterion was total recall (summed across trials 1, 2, 3 and delayed) and the predictors were BNT and LNS scores. These analyses are summarised in Table IV. In the unimpaired controls, BNT $(M=55.42$; $S D=5.09)$ and $\operatorname{LNS}(M=11.10 ; S D=2.65)$ together accounted for $17 \%$ variance in total recall, Multiple $R=.41, F(2,38)=3.91, p=.029$. BNT $(8.8 \%)$ accounted for significant unique variance, but LNS did not.

In the stroke patients with right hemisphere damage only, $\mathrm{BNT}(M=55.50 ; S D=$ $5.22)$ and LNS $(M=9.65 ; S D=3.12)$ together accounted for $68 \%$ variance in total recall, Multiple $R=.83, F(2,19)=18.25, p<.001$. BNT $(55.8 \%)$ accounted for significant unique variance, but LNS did not.

For stroke patients with left hemisphere damage, BNT $(M=51.14 ; S D=7.32)$ and LNS $(M=8.00 ; S D=3.85)$ together accounted for $53 \%$ variance in total recall, Multiple $R=$ $.73, F(2,18)=10.28, p=.001$. BNT $(13.40 \%)$ and LNS $(16.73 \%)$ each accounted for significant unique variance. Thus, LNS was a significant predictor of HVLT-R recall only for the patients with left hemisphere damage.

Insert Table IV here 
In the second set of analyses, the criterion was recognition and the predictors were BNT scores and LNS scores. These analyses are summarised in Table V. In the unimpaired participants, BNT and LNS together accounted for 27.7\% variance in HVLT recognition, Multiple $R=.53, F(2,38)=7.30, p=.002$. BNT $(19.1 \%)$ accounted for significant unique variance, but LNS did not.

For stroke patients with right hemisphere damage only, BNT and LNS together accounted for $61.7 \%$ variance in HVLT recognition, Multiple $R=.785, F(2,17)=13.68, p<$ .001 . BNT (61.6\%) accounted for significant unique variance, but LNS did not.

For stroke patients with left hemisphere damage, BNT and LNS together accounted for $45.4 \%$ variance in recognition, Multiple $R=.67, F(2,18)=7.50, p=.004$. LNS $(24.8 \%)$ accounted for significant unique variance, but BNT did not. Thus, LNS was a significant predictor of HVLT-R recognition only for patients with left hemisphere damage.

\section{Insert Table $\mathrm{V}$}

\section{Discussion}

Relative to stroke patients with damage to the right hemisphere only, those with left hemisphere damage had poorer overall recall, but recognition performance was comparable. This replicates and extends the finding of Schouten et al.'s research [6] in which patients were assessed one year after their stroke. Given that the average time since stroke occurrence was 7.25 years in the current study, our findings suggest that the impairment is long-lasting rather than short-lived. Stroke patients with right hemisphere damage only did not differ significantly from the unimpaired control participants. Of course, it remains possible that 
significant impairment would be observed if the tests were administered closer to the time of stroke.

Relative to unimpaired controls, patients with left hemisphere damage performed more poorly on the immediate recall, delayed recall and recognition tests of the HVLT-R and they showed poorer learning (i.e., less improvement in recall across trials). This suggests that their deficit reflects inadequate encoding rather than impaired retrieval. It is generally agreed that recognition tests impose minimal retrieval demands, whereas free recall tests impose higher retrieval demands $[10 ; 17]$. If the difficulty lay primarily with retrieval, then we might expect impaired recall but intact recognition. In contrast, if the difficulty lay primarily with encoding, then impaired recall and impaired recognition would be expected, because both depend on encoding [17]. The observed group differences are more consistent with the latter than with the former pattern. The finding that patients with left hemisphere damage showed no significant improvement in recall from trial 2 to trial 3, while significant improvement was observed in the unimpaired participants and the patients with right hemisphere damage only is also consistent with the interpretation that left hemisphere stroke patients experienced difficulty forming a durable representation of the verbal material. This encoding difficulty interpretation is further strengthened by the finding that the groups did not differ significantly in terms of the retention index. Thus the ability to maintain the material that was successfully encoded by trial 3 until the delayed recall test, appears to be unaffected by left hemisphere stroke.

The results of the regression analyses predicting HVLT-R performance in unimpaired participants were broadly consistent with expectations based on the encoding and/or retrieval demands imposed by the HVLT-R, LNS and BNT and previous research identifying the brain regions on which these processes rely. That is, BNT (but not LNS) was a significant predictor of HVLT-R recall and recognition performance, suggesting that unimpaired 
participants relied mainly on temporal rather than frontal regions of the left hemisphere.

Stroke patients with right hemisphere damage only showed the same pattern, suggesting they approached the verbal learning and memory task in essentially the same way as the unimpaired participants.

However, for stroke patients with left-hemisphere damage both BNT and LNST were significant predictors of recall, and LNS (but not BNT) was a significant predictor of recognition performance. These findings suggest that stroke patients with left hemisphere damage recruited more diverse regions within the left hemisphere in learning and memorizing verbal materials. Specifically, these additional regions appear to include the left frontal and parietal regions that underpin working memory [8]. Use of working memory resources might represent a posterior-anterior shift as an attempt to compensate for loss of function associated with damage to other left hemisphere regions [13]. The current findings suggest that this strategy did not fully compensate because a significant impairment in verbal learning and memory was still evident in stroke patients with left hemisphere damage. Further research using larger samples and with more detailed information about the extent and precise location of the damaged regions would help us to better understand the role of working memory during verbal learning and memory in the HVLT-R and similar tasks.

Future research might also examine the extent to which the effects of left hemisphere stroke as observed in the current study might be reduced through rehabilitation. As noted above, we had no information regarding the extent of the stroke patients' engagement in rehabilitation over the years since their discharge from hospital. However, given that such services are not easily accessible in Australia, it seems safe to assume that few if any of the stroke patients would have received such therapies on an ongoing basis, and that there would be no systematic difference between the stroke groups in this regard. 
The research findings have practical implications. To the extent that people with left hemisphere damage are forced to use their working memory, everyday tasks that require verbal learning and memory will be more effortful for these individuals than for those with intact left hemispheres. It is important for clinicians and family members to recognise the effortful nature of verbal learning and memory in these individuals and to make the necessary adjustments and allowances. 


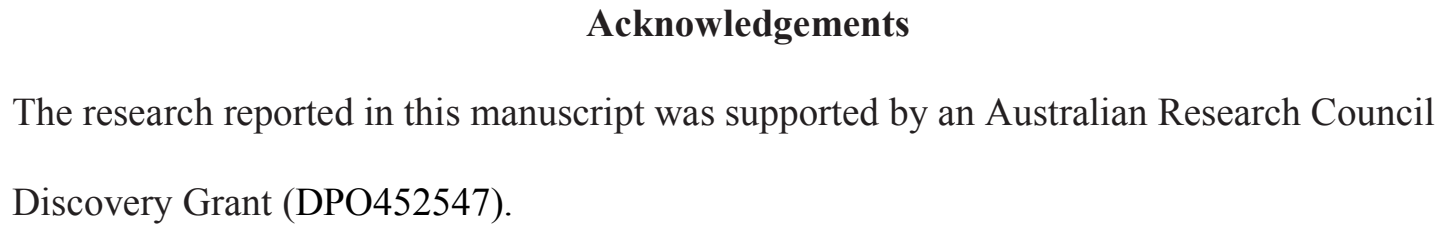

The research reported in this manuscript was supported by an Australian Research Council Discovery Grant (DPO452547).

\section{Declarations of interest}

The authors report no declarations of interest. 


\section{References}

[1] Brandt, J., \& Benedict R. H. (2001). Hopkins Verbal Learning Test-Revised. Lutz (FL): Psychological Assessment Resources.

[2] Frank R. M, Byrne G. J. (2000). The clinical utility of the Hopkins Verbal Learning Test as a screening test for mild dementia. International Journal of Geriatric Psychiatry, $15,317-24$.

[3] Shapiro, A. M., Benedict, R. H. B., Schretlen D., \& Brandt, J., (1999). Construct and Concurrent Validity of the Hopkins Verbal Learning Test - Revised, The Clinical Neuropsychologist, 13, 348-358.

[4] Squire, L.R. (1992). Memory and the hippocampus: a synthesis from findings with rats, monkeys, and humans. Psychological Review, 99, 195-231.

[5] Golby, A. J., Poldrack, R. A., Brewer, J. B., Spencer, D., Desmond, J. E., Aron, A. P., \& Gabrieli, J. D. E. (2001). Material-specific lateralization in the medial temporal lobe and prefrontal cortex during memory encoding. Brain, 124, 1841-1854.

[6] Schouten, E. A., Schiemanck, S. K., Brand, N., \& Post, M. W. M. (2009). Long-term deficits in episodic memory after ischemic stroke: Evaluation and prediction of verbal and visual memory performance based on lesion characteristics. Journal of Stroke and Cerebrovascular Diseases, 18, 128-138.

[7] The Psychological Corporation (1998). Wechsler Adult Intelligence Scale- 3rd Edition Australian Adaptation. Harcourt Brace \& Company (Australia) Pty Ltd.

[8] D’Esposito, M., Aguirre, G. K., Zarahn, D., Ballard, D., Shin, R. K., \& Lease, J. (1998). Functional MRI studies of spatial and nonspatial working memory. Cognitive Brain Research, 7, 1-13.

[9] Hoppenbrouwers, S. S., De Jesus, D. R., Stirpe, T., Fitzgerald, P. B., Voineskos, A. N., Schutter, D. J. L. G., \& Daskalakis, Z. J. (2012). Inhibitory deficits in the dorsolateral 
prefrontal cortex in psychopathic offenders, Cortex, http://dx.doi.org/10.1016/j.cortex.2012.06.003

[10] Bower, G. H., Clark, M. C., Lesgold, A. M., and Winzenz, D. (1969). Hierarchical retrieval schemes in recall of categorized word lists. Journal of Verbal Learning and Verbal Behavior, 8, 323-34.

[11] Kaplan, E., Goodglass, H., \& Weintraub, S. (2001). Boston Naming Test. $2^{\text {nd }}$ Ed. Philadelphia: Lippincott, Williams, \& Wilkins.

[12] Baldo, J. V., Are'valo, A., Patterson, J. P., \& Dronkers, N. F. (2012). Grey and white matter correlates of picture naming: Evidence from a voxel based lesion analysis of the Boston Naming Test, Cortex, doi:10.1016/j.cortex.2012.03.001.

[13] Bangen, K. J., Kaupe, A. R., Mirzakhanian, H., Wierenga, C. E., Jeste, D. V., \& Eyler, L. T. (2012). Compensatory brain activity during encoding among older adults with better recognition memory for face-name pairs: An integrative functional, structural and perfusion imaging study. Journal of the International Neuropsychological Society, 18, 402-413.

[14] Nelson, H. E., (1991). National Adult Reading Test: Second Edition. London: nferNelson Publishing Company Ltd.

[15] Carey, J. R., Evans, C. D., Anderson, D. C., \& Bhatt, E. (2008). Safety of 6-Hz primed low-frequency rTMS in stroke. Neurorehabilitation and Neural Repair, 22, 195-192.

[16] Skidmore, E. R., Whyte, E. M., Holm, M. B., Becker, J. T., Butters, M. A., Dew, M. A., Munin, M. C,. Lenze, E. J. (2010). Cognitive and affective predictors of rehabilitation participation after Stroke. Archives of Phys Med Rehabilitation, 91, 203-207.

[17] Baddeley, A., Eysenck, M. W., \& Anderson, M. C. (2009). Memory. Hove, East Essex: Psychology Press. 
Verbal learning and memory following stroke

Table I.

Demographic details and ability measures for the unimpaired controls, stroke patients with right hemisphere $(\mathrm{RH})$ damage only and stroke patients with left hemisphere $(\mathrm{LH})$ damage

\begin{tabular}{lccc}
\hline & & \multicolumn{2}{c}{ Stroke Groups } \\
\cline { 2 - 4 } & Unimpaired & RH damage only & LH damage \\
\hline$n$ & 41 & 20 & 21 \\
Gender ratio (Female : Male) & $17: 24$ & $8: 12$ & $8: 13$ \\
Mean age years (SD) & $68.02(12.12)$ & $63.45(14.35)$ & $66.71(9.49)$ \\
Years of Education $(S D)$ & $11.71(3.09)$ & $11.95(2.86)$ & $11.14(3.18)$ \\
National Adult Reading Test $(S D)$ & $118.87(7.80)$ & $114.20(7.47)$ & $109.85(9.38)$ \\
Picture Completion $(S D)$ & $18.29(3.99)$ & $16.15(3.69)$ & $16.43(4.68)$
\end{tabular}

URL: http://mc.manuscriptcentral.com/tbin 
Table II.

Time since stroke, and lesion location in stroke patients with right hemisphere (RH) damage only and with left hemisphere (LH) damage.

\begin{tabular}{|c|c|c|}
\hline & \multicolumn{2}{|c|}{ Stroke Group } \\
\hline & RH damage only & LH damage \\
\hline Time since stroke in months $(S D)$ & $84(59)$ & $89(65)$ \\
\hline Anterior & 11 & 15 \\
\hline Posterior & 13 & 15 \\
\hline Subcortical & 14 & 9 \\
\hline
\end{tabular}

URL: http://mc.manuscriptcentral.com/tbin 
Table III.

Mean (SE) HVLT-R recall on trials 1, 2, 3 and delayed, learning, retention, recognition and discrimination indices in the unimpaired controls (n $=41)$, stroke patients with right hemisphere $(R H)$ damage only $(n=20)$ and stroke patients with left hemisphere $($ LH $)$ damage $(n=21)$.

\begin{tabular}{|c|c|c|c|c|c|c|c|c|}
\hline \multirow[b]{2}{*}{ Group } & \multicolumn{4}{|c|}{ Recall Trial } & \multicolumn{4}{|c|}{ HVLT index } \\
\hline & 1 & 2 & 3 & Delayed & Learning & Retention & Recognition & Discrimination \\
\hline Unimpaired & $6.12(0.32)$ & $8.66(0.36)$ & $9.88(0.35)$ & $9.12(0.43)$ & $3.88(.28)$ & $91.29(3.87)$ & $23.27(0.19)$ & $11.27(.20)$ \\
\hline RH damage only & $5.80(0.46)$ & $8.20(0.51)$ & $9.10(0.51)$ & $8.50(0.61)$ & $3.45(.40)$ & $92.07(5.54)$ & $22.85(0.28)$ & $10.80(.29)$ \\
\hline LH damage & $5.05(0.45)$ & $7.00(0.50)$ & $7.48(0.49)$ & $6.43(0.59)$ & $2.81(.39)$ & $87.04(5.41)$ & $22.52(0.27)$ & $10.52(.28)$ \\
\hline
\end{tabular}


Verbal learning and memory following stroke

Table IV.

Results of standard multiple regressions predicting total recall on HVLT-R from BNT and

LNS. BNT was a significant predictor in all groups. LNS was a significant predictor only for stroke patients with left hemisphere (LH) damage.

\begin{tabular}{|c|c|c|c|c|c|c|}
\hline & 1 & 2 & 3 & $B$ & $\beta$ & $s r^{2}$ (unique) \\
\hline \multicolumn{7}{|l|}{ Unimpaired controls } \\
\hline \multicolumn{7}{|l|}{ 1. Total recall } \\
\hline 2. Boston Naming Test & $.37 * *$ & & & .46 & .31 & $.088 *$ \\
\hline 3. Letter Number Sequencing & $.29 *$ & $.31 *$ & & .54 & .19 & .032 \\
\hline \multicolumn{7}{|c|}{ Stroke patients with RH damage only } \\
\hline \multicolumn{7}{|l|}{ 1. Total recall } \\
\hline 2. Boston Naming Test & $.80 * * *$ & & & 1.28 & .76 & $.558 * *$ \\
\hline 3. Letter Number Sequencing & .35 & .20 & & .57 & .20 & .040 \\
\hline \multicolumn{7}{|l|}{ Stroke patients with LH damage } \\
\hline \multicolumn{7}{|l|}{ 1. Total recall } \\
\hline 2. Boston Naming Test & $.61 * *$ & & & .52 & .41 & $.134 *$ \\
\hline 3. Letter Number Sequencing & $.63 * *$ & $.44 *$ & & 1.10 & .46 & $.167 *$ \\
\hline
\end{tabular}

$* p<.05 ; * * p<.01 ; * * * p<.001$ 
Verbal learning and memory following stroke

Table V.

Results of standard multiple regressions predicting HVLT-R recognition from BNT and LNS.

BNT was a significant predictor for the unimpaired participants and for stroke patients with

right hemisphere (RH) damage only. LNS was a significant predictor for stroke patients with

left hemisphere (LH) damage.

\begin{tabular}{|c|c|c|c|c|c|}
\hline$P$ & 1 & 2 & $B$ & $\beta$ & $s r^{2}$ (unique) \\
\hline \multicolumn{6}{|l|}{ Unimpaired controls } \\
\hline \multicolumn{6}{|l|}{ 1. Recognition } \\
\hline 2. Boston Naming Test & $.51 * * *$ & & .10 & .46 & $.191 * *$ \\
\hline 3. Letter Number Sequencing & $.29 *$ & $.31 *$ & .06 & .15 & .021 \\
\hline \multicolumn{6}{|c|}{ Stroke patients with RH damage only } \\
\hline 1. Recognition & & & & & \\
\hline 2. Boston Naming Test & $.77 * * *$ & & .21 & .80 & $.616 * * *$ \\
\hline 3. Letter Number Sequencing & -.01 & .20 & -.08 & -.17 & .027 \\
\hline \multicolumn{6}{|l|}{ Stroke patients with LH damage } \\
\hline \multicolumn{6}{|l|}{ 1. Recognition } \\
\hline 2. Boston Naming Test & $.46^{*}$ & & .04 & .21 & .036 \\
\hline 3. Letter Number Sequencing & $.65 * *$ & $.44 *$ & .19 & .55 & $.248 * *$ \\
\hline
\end{tabular}

$* p<.05 ; * * p<.01 ; * * * p<.001$

URL: http://mc.manuscriptcentral.com/tbin 